\title{
On the Cayley Factorization of Calotte Conditions
}

\author{
Tiong-Seng Tay \\ Department of Mathematics, National University of Singapore, \\ 10 Kent Ridge Cresent, Singapore 0511 \\ mattayts@nuscc.nus.sg
}

\begin{abstract}
The calotte condition is the projective condition that a cycle of lines radiating from the vertices of a plane $n$-gon be the correct projection of a ring of faces surrounding an $n$-gonal piece of plane in space, the spatial figure being not entirely coplanar. This condition can be expressed as a homogeneous bracket polynomial. In general, this polynomial is not Cayley factorable. Henry Crapo conjectured that it becomes so when multiplied by a product of $n-4$ brackets. It is the purpose of this article to prove this conjecture.
\end{abstract}

\section{Introduction}

An important problem in computer-aided geometric reasoning is to find geometric interpretations for algebraic expressions automatically. For projective geometry, invariants can be expressed as homogeneous bracket polynomials. However, it is usually not easy to extract geometric information from a bracket polynomial. On the other hand, the two operations in Cayley algebra, meet and join, correspond to intersection and span. Thus the question reduces to the Cayley factorization problem-expressing a homogeneous bracket polynomial as a Cayley algebra expression (using only the join and meet operations).

White (1991) has an algorithm to do this whenever possible for the multilinear case. (Recall that a bracket polynomial is multilinear if and only if each variable point occurs exactly once in each monomial term which is a product of brackets.)

For the nonmultilinear case, no algorithm exists. However, Sturmfels and Whiteley (1991) proved that a homogeneous bracket polynomial is Cayley factorable after multiplication by a certain number of monomials. Crapo (1991) investigated the nonmultilinear case for several important examples drawn from scene analysis. One of them is the "calotte condition." An $n$-ring is a cycle of $n$ lines $a_{1} b_{1}, a_{2} b_{2}, \ldots, a_{n} b_{n}$ radiating from the vertices $a_{1}, a_{2}, \ldots, a_{n}$ of an $n$-gon in the 


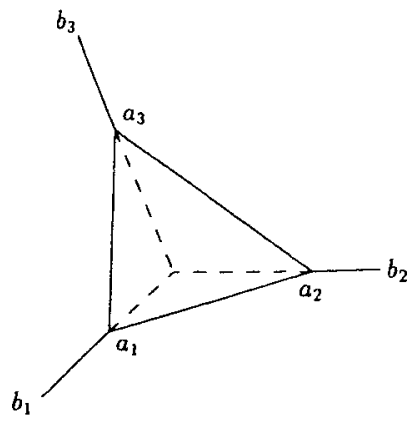

Fig. 1.1. A 3-ring.

plane. This $n$-ring is called an $n$-calotte if it is the correct projection of a ring of faces surrounding an $n$-gonal piece of plane in space, the spatial figure being not entirely coplanar. The projective condition for an $n$-ring to be an $n$-calotte is called the calotte condition. The calotte condition for the 3-ring in Fig. 1.1 is

$$
\left[a_{1} a_{2} b_{2}\right]\left[a_{2} a_{3} b_{3}\right]\left[a_{3} a_{1} b_{1}\right]-\left[b_{1} a_{1} a_{2}\right]\left[b_{2} a_{2} a_{3}\right]\left[b_{3} a_{3} a_{1}\right]=0,
$$

which factorizes to

$$
\left[a_{1} a_{2} a_{3}\right]\left(a_{1} b_{1} \wedge a_{2} b_{2} \wedge a_{3} b_{3}\right)=0
$$

From the latter condition, it is easily seen that the calotte condition is satisfied when either the triangle $a_{1} a_{2} a_{3}$ is collinear or the lines $a_{1} b_{1}, a_{2} b_{2}, a_{3} b_{3}$ are concurrent.

The calotte condition for the 4-ring in Fig. 1.2 is

$$
\left[a_{1} a_{2} b_{2}\right]\left[a_{2} a_{3} b_{3}\right]\left[a_{3} a_{4} b_{4}\right]\left[a_{4} a_{1} b_{1}\right]-\left[b_{1} a_{1} a_{2}\right]\left[b_{2} a_{2} a_{3}\right]\left[b_{3} a_{3} a_{4}\right]\left[b_{4} a_{4} a_{1}\right]=0
$$

This has the following two equivalent Cayley factorizations:

$$
\left(a_{1} b_{1} \wedge a_{2} b_{2}\right) \vee\left(a_{1} a_{4} \wedge a_{2} a_{3}\right) \vee\left(a_{3} b_{3} \wedge a_{4} b_{4}\right)=0
$$

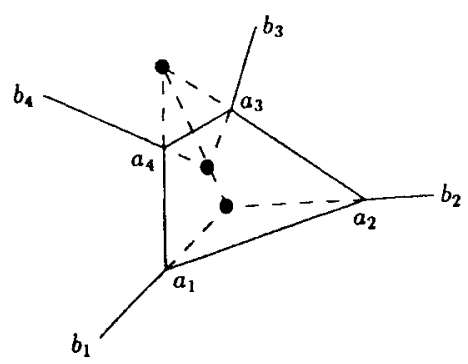

Fig. 1.2. A 4-ring. 
and

$$
\left(a_{1} b_{1} \wedge a_{4} b_{4}\right) \vee\left(a_{2} b_{2} \wedge a_{3} b_{3}\right) \vee\left(a_{1} a_{2} \wedge a_{4} a_{3}\right)=0
$$

which can be interpreted as the three points $\left(a_{1} b_{1} \wedge a_{2} b_{2}\right),\left(a_{1} a_{4} \wedge a_{2} a_{3}\right)$, $\left(a_{3} b_{3} \wedge a_{4} b_{4}\right)$ and the three points $a_{1} b_{1} \wedge a_{4} b_{4}, a_{2} b_{2} \wedge a_{3} b_{3}, a_{1} a_{2} \wedge a_{4} a_{3}$ being collinear, respectively.

The calotte condition for the 5-ring is not Cayley factorizable but becomes so when multiplied by a single bracket $\left[a_{1} a_{3} a_{4}\right]$.

The above Cayley factorizations can be found in [1]. Crapo also conjectured that the calotte condition for an $n$-ring, $n \geq 5$, is not Cayley factorizable but becomes so when multiplied by $n-4$ brackets. It is the purpose of this paper to prove the second part of this conjecture.

Since we are concerned with Cayley factorization, the points are assumed to be in generic position. More precisely, we assume that the coordinates of the points are independent indeterminates over the reals. The bracket $[a b c]$ is then a polynomial whose variables are the coordinates of $a, b$, and $c$.

We now give a brief description of Cayley algebra. Let $V$ be a $d$-dimensional vector space over a field $K$. In our case $V=\mathbb{R}[X]^{3}$, where $\mathbb{R}[X]$ is the vector space of polynomials with real coefficients, whose variables are the homogeneous coordinates, $\left(x_{1}, x_{2}, x_{3}\right)$, of points of the calotte in question. Let $\wedge V$ denote the exterior algebra on $V$. The exterior product, known as the join, of $a, b$ is denoted by $a \vee b$ or simply $a b$. Note that the join of $a_{1}, \ldots, a_{k}$ is nonzero if and only if the $a_{i}$ 's are distinct and linearly independent. The join of $d$ points $a_{1}, \ldots, a_{d}$, denoted by the bracket $\left[a_{1}, \ldots, a_{d}\right]$, is the determinant of the vectors $a_{1}, \ldots, a_{d}$.

The second operation meet $(\wedge)$ is defined as follows. If $A=a_{1} \cdots a_{j}, B=b_{1} \cdots b_{k}$ with $j+k \geq d$, then

$$
A \wedge B=\sum_{\sigma} \operatorname{sign}(\sigma)\left[a_{\sigma(1)}, \ldots, a_{\sigma(d-k)}, b_{1}, \ldots, b_{k}\right] a_{\sigma(d-k+1)} \cdots a_{\sigma(j)}
$$

The sum is taken over all permutations $\sigma$ of $\{1,2, \ldots, j\}$ such that

$$
\sigma(1)<\sigma(2)<\cdots<\sigma(d-k) \text { and } \sigma(d-k+1)<\sigma\left(d_{k}+2\right)<\cdots<\sigma(j) \text {. }
$$

The meet is associative, distributive over addition and anticommutative in the sense that $A \wedge B=(-1)^{(d-k)(d-j)} B \wedge A$. In our case we work with the real projective plane, so $d=3$. Thus $a b \wedge c d=[a c d] b-[b c d] a=[a b d] c-[a b c] d$.

\section{Pointing Algorithm}

A closer examination of the condition for the 4-ring reveals the following. If we let $x=a_{1} a_{4} \wedge a_{2} a_{3}$ and $y=a_{3} b_{3} \wedge a_{4} b_{4}$ to obtain the 3-ring in Fig. 2.1, then the calotte condition for the 4-ring is equivalent to the calotte condition for this 3 -ring. 


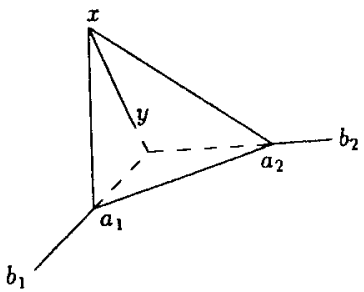

Fig. 2.1. A 3-ring.

This procedure of reduction is called pointing. In general this procedure can be used to reduce an $n$-calotte to an $(n-1)$-calotte with an almost equivalent condition. In this section we look at one pointing sequence which allows us to get a proof of the main theorem.

For the $n$-ring shown in Fig. 2.2, the calotte condition is

$$
\begin{aligned}
C(n)= & {\left[a_{1} a_{2} b_{2}\right]\left[a_{2} a_{3} b_{3}\right] \cdots\left[a_{n-1} a_{n} b_{n}\right]\left[a_{n} a_{1} b_{1}\right] } \\
& -\left[b_{1} a_{1} a_{2}\right]\left[b_{2} a_{2} a_{3}\right] \cdots\left[b_{n-1} a_{n-1} a_{n}\right]\left[b_{n} a_{n} a_{1}\right]=0 .
\end{aligned}
$$

Let

$$
\begin{aligned}
x_{n-1} & =a_{1} a_{n} \wedge a_{n-2} a_{n-1}, & y_{n-1} & =a_{n-1} b_{n-1} \wedge a_{n} b_{n}, \\
x_{n-2} & =a_{1} x_{n-1} \wedge a_{n-3} a_{n-2}, & y_{n-2} & =a_{n-2} b_{n-2} \wedge x_{n-1} y_{n-1}, \\
& \vdots & & \vdots \\
x_{m} & =a_{1} x_{m+1} \wedge a_{m-1} a_{m}, & y_{m} & =a_{m} b_{m} \wedge x_{m+1} y_{m+1}, \\
& \vdots & & \vdots \\
x_{4} & =a_{1} x_{5} \wedge a_{3} a_{4}, & y_{4} & =a_{4} b_{4} \wedge x_{5} y_{5} .
\end{aligned}
$$

$\left\{x_{n-1}, y_{n-1}, \ldots, x_{4}, y_{4}\right\}$ is call a pointing sequence. Then the derived $m$-ring corresponding to this pointing sequence is the calotte with vertices $a_{1}, a_{2}, \ldots, a_{m-1}, x_{m}$

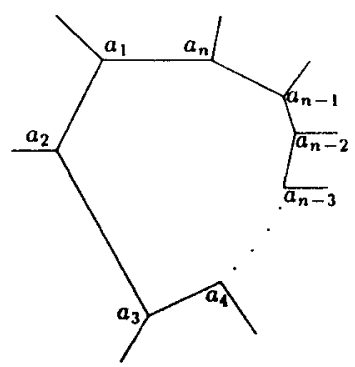

Fig. 2.2. An $n$-ring. 


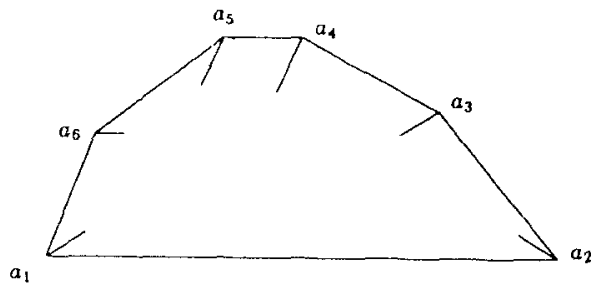

(a)

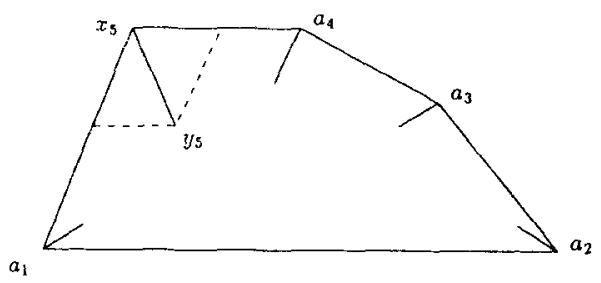

(b)

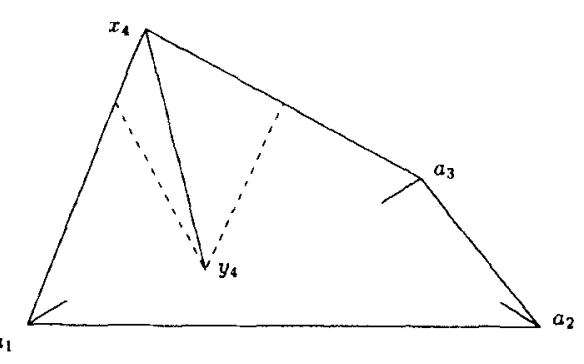

(c)

Fig. 2.3. A 6-ring (a) and its derived 5-ring (b) and 4-ring (c).

and $b_{1}, b_{2}, \ldots, b_{m-1}, y_{m}$. Figure 2.3 shows a 6-ring together with its derived 5 - and 4-rings.

Let

$$
\begin{aligned}
C\left(m^{\prime}\right)= & {\left[a_{1} a_{2} b_{2}\right]\left[a_{2} a_{3} b_{3}\right] \cdots\left[a_{m-2} a_{m-1} b_{m-1}\right]\left[a_{m-1} x_{m} y_{m}\right]\left[x_{m} a_{1} b_{1}\right] } \\
& -\left[b_{1} a_{1} a_{2}\right]\left[b_{2} a_{2} a_{3}\right] \cdots\left[b_{m-2} a_{m-2} a_{m-1}\right]\left[b_{m-1} a_{m-1} x_{m}\right]\left[y_{m} x_{m} a_{1}\right]
\end{aligned}
$$

Then $C\left(m^{\prime}\right)=0$ is the condition for the derived $m$-ring

Theorem 2.1. If $n>4$, then

$$
\begin{aligned}
C\left((n-1)^{\prime}\right)= & {\left[a_{1} a_{n-2} a_{n-1}\right]\left[a_{1} a_{n-2} a_{n}\right] C(n), } \\
C\left(4^{\prime}\right)= & {\left[a_{1} a_{n-2} a_{n-1}\right]\left[a_{1} a_{n-2} a_{n}\right]\left[a_{1} a_{n-3} a_{n-2}\right]\left[a_{1} a_{n-3} x_{n-1}\right] } \\
& \times\left[a_{1} a_{n-4} a_{n-3}\right]\left[a_{1} a_{n-4} x_{n-2}\right] \cdots\left[a_{1} a_{3} a_{4}\right]\left[a_{1} a_{3} x_{5}\right] C(n) .
\end{aligned}
$$

Proof. First note that

$$
\begin{aligned}
C(n)= & {\left[a_{1} a_{2} b_{2}\right]\left[a_{2} a_{3} b_{3}\right] \cdots\left[a_{n-1} a_{n} b_{n}\right]\left[a_{n} a_{1} b_{1}\right] } \\
& -\left[b_{1} a_{1} a_{2}\right]\left[b_{2} a_{2} a_{3}\right] \cdots\left[b_{n-1} a_{n-1} a_{n}\right]\left[b_{n} a_{n} a_{1}\right]
\end{aligned}
$$


and

$$
\begin{aligned}
C\left((n-1)^{\prime}\right)= & {\left[a_{1} a_{2} b_{2}\right]\left[a_{2} a_{3} b_{3}\right] \cdots\left[a_{n-3} a_{n-2} b_{n-2}\right]\left[a_{n-2} x_{n-1} y_{n-1}\right]\left[x_{n-1} a_{1} b_{1}\right] } \\
& -\left[b_{1} a_{1} a_{2}\right]\left[b_{2} a_{2} a_{3}\right] \cdots\left[b_{n-3} a_{n-3} a_{n-2}\right]\left[b_{n-2} a_{n-2} x_{n-1}\right]\left[y_{n-1} x_{n-1} a_{1}\right] .
\end{aligned}
$$

So it suffices to prove that

$$
\begin{aligned}
& {\left[b_{n-2} a_{n-2} x_{n-1}\right]\left[y_{n-1} x_{n-1} a_{1}\right]} \\
& \quad=\left[b_{n-2} a_{n-2} a_{n-1}\right]\left[b_{n-1} a_{n-1} a_{n}\right]\left[b_{n} a_{n} a_{1}\right]\left[a_{1} a_{n-2} a_{n-1}\right]\left[a_{1} a_{n-2} a_{n}\right]
\end{aligned}
$$

and

$$
\begin{aligned}
& {\left[a_{n-2} x_{n-1} y_{n-1}\right]\left[x_{n-1} a_{1} b_{1}\right]} \\
& \quad=\left[a_{n-2} a_{n-1} b_{n-1}\right]\left[a_{n-1} a_{n} b_{n}\right]\left[a_{n} a_{1} b_{1}\right]\left[a_{1} a_{n-2} a_{n-1}\right]\left[a_{1} a_{n-2} a_{n}\right] .
\end{aligned}
$$

Since

$$
\begin{aligned}
& x_{n-1}=a_{1} a_{n} \wedge a_{n-2} a_{n-1}, \\
& y_{n-1}=a_{n-1} b_{n-1} \wedge a_{n} b_{n},
\end{aligned}
$$

we have

$$
\begin{aligned}
x_{n-1} & =\left[a_{1} a_{n} a_{n-1}\right] a_{n-2}-\left[a_{1} a_{n} a_{n-2}\right] a_{n-1} \\
& =\left[a_{1} a_{n-2} a_{n}\right] a_{n-1}-\left[a_{1} a_{n-1} a_{n}\right] a_{n-2}
\end{aligned}
$$

So

$$
\left[b_{n-2} a_{n-2} x_{n-1}\right]=\left[a_{1} a_{n-2} a_{n}\right]\left[b_{n-2} a_{n-2} a_{n-1}\right] .
$$

Also

$$
\begin{aligned}
& x_{n-1}=\left[a_{1} a_{n-2} a_{n-1}\right] a_{n}-\left[a_{n-2} a_{n-1} a_{n}\right] a_{1}, \\
& y_{n-1}=\left[a_{n-1} b_{n-1} b_{n}\right] a_{n}-\left[a_{n-1} b_{n-1} a_{n}\right] b_{n} .
\end{aligned}
$$

Therefore

$$
\left[y_{n-1} x_{n-1} a_{1}\right]=\left[a_{n-2} a_{n-1} a_{1}\right]\left[b_{n-1} a_{n-1} a_{n}\right]\left[b_{n} a_{n} a_{1}\right]
$$

This proves (2.1). Equation (2.2) can be proved similarly.

The second equality follows by an inductive argument. 


\section{An Example}

We now illustrate the proof of the main proof using the case $n=6$. The proof of the general case works along the same reasoning and is presented in the next section.

According to Theorem 2.1,

$$
C\left(4^{\prime}\right)=\left[a_{1} a_{4} a_{5}\right]\left[a_{1} a_{4} a_{6}\right]\left[a_{1} a_{3} a_{4}\right]\left[a_{1} a_{3} x_{5}\right] C(6)
$$

where

$$
\begin{aligned}
& x_{5}=a_{1} a_{6} \wedge a_{4} a_{5}, \quad y_{5}=a_{5} b_{5} \wedge a_{6} b_{6}, \\
& x_{4}=a_{1} x_{5} \wedge a_{3} a_{4}, \quad y_{4}=a_{4} b_{4} \wedge x_{5} y_{5} .
\end{aligned}
$$

However,

$$
C\left(4^{\prime}\right)=\left(a_{1} b_{1} \wedge a_{2} b_{2}\right) \vee\left(a_{1} x_{4} \wedge a_{2} a_{3}\right) \vee\left(a_{3} b_{3} \wedge x_{4} y_{4}\right)
$$

The first step is to write $x_{4}, x_{5}, y_{4}, y_{5}$ in terms of the $a_{i}$ 's and $b_{i}$ 's. These are done inductively as follows. Let

$$
\beta_{4}=\left[a_{1} a_{4} a_{5}\right]\left[a_{1} a_{3} a_{4}\right], \quad \beta_{5}=\left[a_{1} a_{4} a_{5}\right]
$$

Thus, from (3.2),

$$
\begin{aligned}
x_{5} & =\left[a_{1} a_{4} a_{5}\right] a_{6}-\left[a_{6} a_{4} a_{5}\right] a_{1}=\alpha_{5} a_{1}+\beta_{5} a_{6}, \\
x_{4} & =\left[a_{1} a_{3} a_{4}\right] x_{5}-\left[x_{5} a_{3} a_{4}\right] a_{1}=\left[a_{1} a_{3} a_{4}\right] \beta_{5} a_{6}+\alpha_{4} a_{6} \\
& =\beta_{4} a_{6}+\alpha_{4} a_{1},
\end{aligned}
$$

where $\alpha_{5}, \alpha_{4}$ are bracket polynomials, and

$$
\left[a_{1} a_{3} x_{5}\right]=\beta_{5}\left[a_{1} a_{3} a_{6}\right]
$$

From (3.4) and (3.7), (3.1) can be rewritten as

$$
C\left(4^{\prime}\right)=\beta_{4} \beta_{5}\left[a_{1} a_{4} a_{6}\right]\left[a_{1} a_{3} a_{6}\right] C(6) .
$$


Next we have, from (3.5) and (3.6),

$$
\begin{aligned}
x_{5} y_{5} & =\left(a_{1} a_{6} \wedge a_{4} a_{5}\right) \vee\left(a_{5} b_{5} \wedge a_{6} b_{6}\right), \\
x_{4} y_{4} & =\left(a_{1} x_{5} \wedge a_{3} a_{4}\right) \vee\left(a_{4} b_{4} \wedge x_{5} y_{5}\right) \\
& =\beta_{5}\left(a_{1} a_{6} \wedge a_{3} a_{4}\right) \vee\left(a_{4} b_{4} \wedge x_{5} y_{5}\right), \\
a_{1} x_{4} & =\beta_{4} a_{1} a_{6} .
\end{aligned}
$$

From (3.9), (3.10), and (3.11), (3.3) can be written as

$$
\begin{aligned}
C\left(4^{\prime}\right)= & \beta_{4} \beta_{5}\left(a_{1} b_{1} \wedge a_{2} b_{2}\right) \vee\left(a_{1} a_{6} \wedge a_{2} a_{3}\right) \\
& \vee\left\{a_{3} b_{3} \wedge\left\{\left(a_{1} a_{6} \wedge a_{3} a_{4}\right) \vee\left(a_{4} b_{4} \wedge\left(\left(a_{1} a_{6} \wedge a_{4} a_{5}\right) \vee\left(a_{5} b_{5} \wedge a_{6} b_{6}\right)\right)\right)\right\}\right\} .
\end{aligned}
$$

Canceling $\beta_{4}$ and $\beta_{5}$ in the two expressions of $C\left(4^{\prime}\right)$ given in (3.8) and (3.12) yields the desired Cayley factorization of $C(6)$ multiplied by two brackets.

\section{Proof of the Main Theorem}

As stated in Section 1, we know that

$$
C\left(4^{\prime}\right)=\left(a_{1} b_{1} \wedge a_{2} b_{2}\right) \vee\left(a_{1} x_{4} \wedge a_{2} a_{3}\right) \vee\left(a_{3} b_{3} \wedge x_{4} y_{4}\right)
$$

We now want to show that when $x_{4}$ and $y_{4}$ are written in terms of the $a$ 's and $b$ 's, this Cayley expression can be written as the product of two factors, one is a Cayley expression while the other is a product of exactly $(n-4)$ brackets called $\beta_{4}, \ldots, \beta_{n-1}$. Theorem 2.1 says that $C\left(4^{\prime}\right)$ is $C(n)$ times $2(n-4)$ brackets. We will show that when $x_{4}$ and $y_{4}$ are substituted, these brackets are the product of the $\beta_{i}$ 's and another $n-4$ brackets. Cancellation will then give a Cayley factorization of $C(n)$ multiplied by $n-4$ brackets.

For $4 \leq m<n$, define

$$
\beta_{m}=\left[a_{1} a_{n-2} a_{n-1}\right]\left[a_{1} a_{n-3} a_{n-2}\right] \cdots\left[a_{1} a_{m-1} a_{m}\right]
$$

and

$$
A_{m}=\left(( a _ { 1 } a _ { n } \wedge a _ { m - 2 } a _ { m - 1 } ) \vee \left(a_{m-1} b_{m-1} .\right.\right.
$$

Note the unbalanced brackets in $A_{m}$. For convenience we assume that $\beta_{n}=1$.

We need to prove three lemmas before presenting the proof of the main theorem. 
Lemma 4.1. For each $m$ such that $4 \leq m \leq n-1, x_{m}=\alpha_{m} a_{1}+\beta_{m} a_{n}$, where $\alpha_{m}$ is a bracket polynomial in the $a_{i}$ 's.

Proof. Clearly, the assertion is true for $m=n-1$ as

$$
x_{n-1}=\left[a_{1} a_{n-2} a_{n-1}\right] a_{n}-\left[a_{n-2} a_{n-1} a_{n}\right] a_{1} .
$$

Suppose the result is true for $m$, then

$$
\begin{aligned}
x_{m-1} & =a_{1} x_{m} \wedge a_{m-2} a_{m-1} \\
& =\left[a_{1} a_{m-2} a_{m-1}\right] x_{m}-\left[x_{m} a_{m-2} a_{m-1}\right] a_{1} \\
& =\beta_{m}\left[a_{1} a_{m-2} a_{m-1}\right] a_{n}+\left(\alpha_{m}\left[a_{1} a_{m-2} a_{m-1}\right]-\left[x_{m} a_{m-2} a_{m-1}\right]\right) a_{1}
\end{aligned}
$$

Since $\beta_{m-1}=\beta_{m}\left[a_{1} a_{m-2} a_{m-1}\right]$, the result follows by induction.

Lemma 4.2. For each $m$ such that $4 \leq m \leq n-1$,

$$
\left[a_{m-2} x_{m} a_{1}\right]=\beta_{m}\left[a_{m-2} a_{n} a_{1}\right]
$$

Lemma 4.3. For each $m$ such that $4 \leq m \leq n-1$,

$$
\left.\left.\left.\left(x_{m} y_{m}\right)=\beta_{m+1} \beta_{m+2} \cdots \beta_{n} A_{m+1} \wedge A_{m+2} \wedge \cdots \wedge A_{n} \wedge a_{n} b_{n}\right)\right) \cdots\right)
$$

Proof. We prove this assertion by induction. Clearly, it is true for $m=n-1$, as

$$
\begin{aligned}
\left(x_{n-1} y_{n-1}\right) & =\left(\left(a_{1} a_{n} \wedge a_{n-2} a_{n-1}\right) \vee\left(a_{n-1} b_{n-1} \wedge a_{n} b_{n}\right)\right) \\
& \left.\left.=\beta_{n} A_{n} \wedge a_{n} b_{n}\right)\right) .
\end{aligned}
$$

Now we assume that the assertion is true for $m+1$ and prove that it is true for $m$. Thus

$$
\begin{aligned}
\left(x_{m} y_{m}\right) & =\left(\left(a_{1} x_{m+1} \wedge a_{m-1} a_{m}\right) \vee\left(a_{m} b_{m} \wedge\left(x_{m+1} y_{m+1}\right)\right)\right) \\
& =\left(\left(a_{1}\left(\alpha_{m+1} a_{1}+\beta_{m+1} a_{n}\right) \wedge a_{m-1} a_{m}\right) \vee\left(a_{m} b_{m} \wedge\left(x_{m+1} y_{m+1}\right)\right)\right) \\
& \left.\left.=\beta_{m+1} A_{m+1} \wedge\left(x_{m+1} y_{m+1}\right)\right)\right) \\
& \left.\left.=\beta_{m+1} \beta_{m+2} \cdots \beta_{n} A_{m+1} \wedge A_{m+2} \wedge \cdots \wedge A_{n} \wedge a_{n} b_{n}\right) \cdots\right)
\end{aligned}
$$

and the proof is complete.

\section{Lemma 4.4.}

$$
\left.\left.C\left(4^{\prime}\right)=\beta_{4} \beta_{5} \cdots \beta_{n-1}\left(a_{1} b_{1} \wedge a_{2} b_{2}\right) \vee A_{4} \wedge A_{5} \wedge \cdots A_{n} \wedge a_{n} b_{n}\right) \cdots\right)
$$


Proof. Since $a_{1} x_{4}=\beta_{4} a_{1} a_{n}$ and

$$
\left.\left.x_{4} y_{4}=\beta_{5} \beta_{6} \cdots \beta_{n} A_{5} \wedge A_{6} \wedge \cdots \wedge A_{n} \wedge a_{n} b_{n}\right) \cdots\right)
$$

we have

$$
\left.\left.C\left(4^{\prime}\right)=\beta_{4} \beta_{5} \cdots \beta_{n-1}\left(a_{1} b_{1} \wedge a_{2} b_{2}\right) \vee A_{4} \wedge A_{5} \wedge \cdots A_{n} \wedge a_{n} b_{n}\right) \cdots\right)
$$

Theorem 4.5. The calotte condition $C(n)=0$ for an $n$-ring, $n \geq 4$, is Cayley factorable after multiplication by $n-4$ factors. In particular,

$$
\begin{aligned}
{\left[a_{1} a_{3} a_{n}\right]\left[a_{1} a_{4} a_{n}\right] \cdots\left[a_{1} a_{n-2} a_{n}\right] C(n) } & \\
= & \left(a_{1} b_{1} \wedge a_{2} b_{2}\right) \vee\left(( a _ { 1 } a _ { n } \wedge a _ { 2 } a _ { 3 } ) \vee \left(a _ { 3 } b _ { 3 } \wedge \left(( a _ { 1 } a _ { n } \wedge a _ { 3 } a _ { 4 } ) \vee \left(a_{4} b_{4} \wedge \cdots\right.\right.\right.\right. \\
& \wedge\left(\left(a_{1} a_{n} \wedge a_{n-2} a_{n-1}\right) \vee\left(a_{n-1} b_{n-1} \wedge a_{n} b_{n}\right) \cdots\right) .
\end{aligned}
$$

Proof. From Lemma 4.4 and the definition of the $A_{n}$ 's, we have

$$
\begin{aligned}
C\left(4^{\prime}\right)= & \beta_{4} \beta_{5} \cdots \beta_{n-1}\left(a_{1} b_{1} \wedge a_{2} b_{2}\right) \vee\left(( a _ { 1 } a _ { n } \wedge a _ { 2 } a _ { 3 } ) \vee \left(a _ { 3 } b _ { 3 } \wedge \left(\left(a_{1} a_{n} \wedge a_{3} a_{4}\right)\right.\right.\right. \\
& \vee\left(a_{4} b_{4} \wedge \cdots \wedge\left(\left(a_{1} a_{n} \wedge a_{n-2} a_{n-1}\right) \vee\left(a_{n-1} b_{n-1} \wedge a_{n} b_{n}\right) \cdots\right) .\right.
\end{aligned}
$$

From Lemma 4.1 we have, for $5 \leq m \leq n-1$,

$$
\left[a_{1} a_{m-2} x_{m}\right]=\beta_{m}\left[a_{1} a_{m-2} a_{n}\right]
$$

and by the definition

$$
\beta_{4}=\left[a_{1} a_{n-2} a_{n-1}\right]\left[a_{1} a_{n-3} a_{n-2}\right] \cdots\left[a_{1} a_{3} a_{4}\right]
$$

Thus, from Theorem 2.1, we have

$$
\begin{aligned}
C\left(4^{\prime}\right)= & {\left[a_{1} a_{n-2} a_{n-1}\right]\left[a_{1} a_{n-2} a_{n}\right]\left[a_{1} a_{n-3} a_{n-2}\right]\left[a_{1} a_{n-3} x_{n-1}\right] } \\
& \times\left[a_{1} a_{n-4} a_{n-3}\right]\left[a_{1} a_{n-4} x_{n-2}\right] \cdots\left[a_{1} a_{3} a_{4}\right]\left[a_{1} a_{3} x_{5}\right] C(n) \\
= & \left(\left[a_{1} a_{n-2} a_{n-1}\right]\left[a_{1} a_{n-3} a_{n-2}\right] \cdots\left[a_{1} a_{3} a_{4}\right]\right) \beta_{5} \beta_{6} \cdots \beta_{n-1} \\
& \times\left[a_{1} a_{3} a_{n}\right]\left[a_{1} a_{4} a_{n}\right] \cdots\left[a_{1} a_{n-3} a_{n}\right]\left[a_{1} a_{n-2} a_{n}\right] C(n) \\
= & \beta_{4} \beta_{5} \cdots \beta_{n-1}\left[a_{1} a_{3} a_{n}\right]\left[a_{1} a_{4} a_{n}\right] \cdots\left[a_{1} a_{n-2} a_{n}\right] C(n) .
\end{aligned}
$$

Since each bracket $\left[a_{1} a_{2} a_{3}\right]$ is a polynomial whose variables are the coordinates of the $a_{i}$ 's, the $\beta_{i}$ 's and $C(n)$ are all polynomials in the coordinates of the points. The same is true of the Cayley expression of $C\left(4^{\prime}\right)$ in Lemma 4.4. Moreover, the $\beta_{i}$ 's are nonzero polynomials. Thus we can cancel the $\beta_{i}$ 's from the two expressions of $C\left(4^{\prime}\right)$ to get the desired identity. 
Remark 4.6. Note that the Cayley factorization gives a geometric condition which corresponds to the calotte condition for the derived calotte obtained from the pointing sequence we used. For example, the Cayley factorization of $C(4)$ gives the condition

$$
\begin{aligned}
0 & =\left(a_{1} b_{1} \wedge a_{2} b_{2}\right) \vee\left(a_{1} a_{4} \wedge a_{2} a_{3}\right) \vee\left(a_{3} b_{3} \wedge a_{4} b_{4}\right) \\
& =\left(a_{1} b_{1} \wedge a_{2} b_{2}\right) \vee\left(x_{3}\right) \vee\left(y_{3}\right) .
\end{aligned}
$$

This is precisely the geometric condition for the derived 3-ring.

\section{Other Pointing Sequences}

In the previous sections we have shown that pointing gives rise to an inductive procedure which reduces an $n$-calotte to a 4 -calotte. Subject to the condition that certain brackets are not zero, we have

$$
C(n)=0 \Leftrightarrow C\left(4^{\prime}\right)=0
$$

We also show that, for a particular pointing sequence, we have a particularly nice result.

However, there are other pointing sequences. For example, if we use the pointing sequence $\left\{x_{5}, y_{5}, x_{4}, y_{4}\right\}$ for the 6-ring in Fig. 5.1, then the calotte condition $C\left(4^{*}\right)$ of the derived 4-ring satisfies

$$
\left[a_{6} a_{2} a_{3}\right]\left[a_{6} a_{1} a_{3}\right]\left[a_{5} x_{5} a_{3}\right]\left[x_{5} a_{4} a_{5}\right] C(6)=C\left(4^{*}\right),
$$

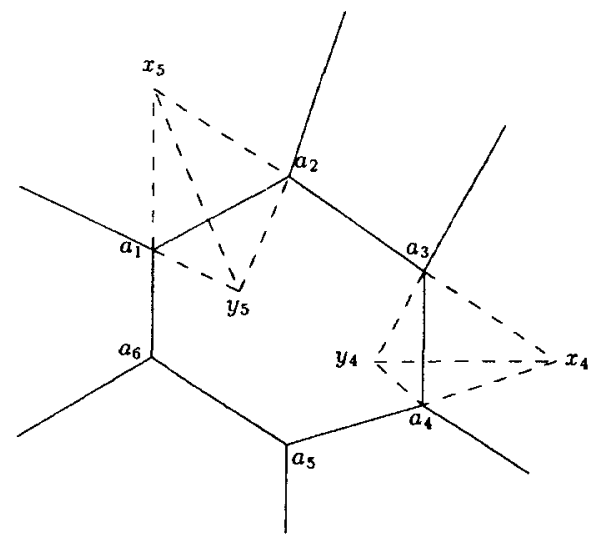

Fig. 5.1. A 6-ring. 
where

$$
\begin{gathered}
x_{5}=a_{1} a_{6} \wedge a_{2} a_{3}=\left[a_{1} a_{6} a_{3}\right] a_{2}-\left[a_{1} a_{6} a_{2}\right] a_{3}=\left[a_{2} a_{3} a_{1}\right] a_{6}-\left[a_{2} a_{3} a_{6}\right] a_{1}, \\
x_{4}=a_{3} x_{5} \wedge a_{4} a_{5}=\left[a_{3} x_{5} a_{4}\right] a_{5}-\left[a_{3} x_{5} a_{5}\right] a_{4}=\left[a_{5} a_{4} a_{3}\right] x_{5}-\left[a_{4} a_{5} x_{5}\right] a_{3}, \\
y_{5}=b_{1} a_{1} \wedge b_{2} a_{2}, \quad y_{4}=b_{3} a_{3} \wedge b_{4} a_{4} .
\end{gathered}
$$

Hence

$$
\begin{aligned}
x_{4} y_{4} & =\left(a_{3} x_{5} \wedge a_{4} a_{5}\right) \vee\left(b_{3} a_{3} \wedge b_{4} a_{4}\right) \\
& =\left[a_{1} a_{6} a_{3}\right]\left(a_{3} a_{2} \wedge a_{4} a_{5}\right) \vee\left(b_{3} a_{3} \wedge b_{4} a_{4}\right), \\
a_{6} x_{5} & =\left[a_{2} a_{3} a_{6}\right] a_{1} a_{6}, \\
x_{4} a_{5} & =-\left[a_{3} x_{5} a_{5}\right] a_{4} a_{5}, \\
{\left[x_{5} a_{4} a_{5}\right] } & =\left[a_{2} a_{3} a_{1}\right]\left[a_{6} a_{4} a_{5}\right]-\left[a_{2} a_{3} a_{5}\right]\left[a_{1} a_{4} a_{5}\right], \\
x_{4} x_{5} & =-\left[x_{5} a_{4} a_{5}\right] a_{3} x_{5}=-\left[a_{1} a_{6} a_{3}\right]\left[x_{5} a_{4} a_{5}\right] a_{3} a_{2}, \\
{\left[a_{5} x_{5} a_{3}\right] } & =\left[a_{1} a_{6} a_{3}\right]\left[a_{5} a_{2} a_{3}\right] .
\end{aligned}
$$

However,

$$
C\left(4^{*}\right)=\left(x_{5} y_{5} \wedge x_{4} y_{4}\right) \vee\left(a_{6} x_{5} \wedge x_{4} a_{5}\right) \vee\left(a_{6} b_{6} \wedge a_{5} b_{5}\right) .
$$

Substitution then gives

$$
\begin{aligned}
&\left(\left[a_{2} a_{3} a_{6}\right]\left[a_{1} a_{4} a_{5}\right]-\left[a_{2} a_{3} a_{1}\right]\left[a_{6} a_{4} a_{5}\right]\right) C(6) \\
&=\left\{\left(\left(a_{2} a_{3} \wedge a_{1} a_{6}\right) \vee\left(b_{1} a_{1} \wedge b_{2} a_{2}\right)\right) \wedge\left(\left(a_{3} a_{2} \wedge a_{4} a_{5}\right) \vee\left(b_{3} a_{3} \wedge b_{4} a_{4}\right)\right)\right\} \\
& \vee\left(a_{1} a_{6} \wedge a_{4} a_{5}\right) \vee\left(a_{6} b_{6} \wedge a_{5} b_{5}\right) .
\end{aligned}
$$

However, there is an equivalent expression for $C\left(4^{*}\right)$ :

$$
C\left(4^{*}\right)=\left(x_{5} y_{5} \wedge a_{6} b_{6}\right) \vee\left(x_{4} y_{4} \wedge a_{5} b_{5}\right) \vee\left(x_{4} x_{5} \wedge a_{5} a_{6}\right) .
$$

From this we have

$$
\begin{aligned}
{\left[a_{6} a_{2} a_{3}\right]\left[a_{5} a_{2} a_{3}\right] C(6)=} & \left\{\left(\left(a_{1} a_{6} \wedge a_{2} a_{3}\right) \vee\left(b_{1} a_{1} \wedge b_{2} a_{2}\right)\right) \wedge a_{6} b_{6}\right\} \\
& \vee\left\{\left(\left(a_{3} a_{2} \wedge a_{4} a_{5}\right) \vee\left(b_{3} a_{3} \wedge b_{4} a_{4}\right)\right) \wedge a_{5} b_{5}\right\} \\
& \vee\left\{a_{3} a_{2} \wedge a_{5} a_{6}\right\}
\end{aligned}
$$


So we see that the calotte condition $C(n)$ can be factorized in many different ways, each corresponding to a pointing sequence. However, other than the particular pointing sequence used in the previous section, in general the multiplier need not be a monomial, i.e., a product of brackets. However, we believe that the monomial terms of the bracket polynomial used as the multiplier are products of $n-4$ brackets.

\section{Acknowledgment}

We would like to thank the referees for some helpful comments.

\section{References}

1. H. Crapo: Invariant-theoretic methods in scene analysis and structural mechanics, Journal of Symbolic Computation 11(5-6) (1991), 523-548.

2. B. Sturmfels and W. Whiteley: On the synthetic factorization of homogeneous invariants, Journal of Symbolic Computation 11(5-6) (1991), 439-454.

3. N. L. White: Multilinear Cayley factorization, Journal of Symbolic Computation 11(5-6) (1991), $421-438$.

Received February 13, 1992, and in revised form November 12, 1992. 